\title{
Perfil de pacientes estomizados: revisão integrativa da literatura
}

\section{Profile of ostomy patients: integrative review of the literature}

\section{Perfil de los pacientes ostomizados: revisión integrativa de la literatura}

${ }^{1}$ Alyne Leal de Alencar Luz; ${ }^{2}$ Maria Helena Barros Araújo Luz ; ${ }^{3}$ Arthur Antunes; ${ }^{4}$ Gilvan Santos de Oliveira;

${ }^{5}$ Elaine Maria Leite Rangel Andrade; ${ }^{6}$ Sara Machado Miranda.

\author{
${ }^{1,6}$ Enfermeira. Mestranda do Programa de Pós-graduação Mestrado em Enfermagem da \\ Universidade Federal do Piauí -UFPI. E-mail: alyne-luz@bol.com.br \\ ${ }^{2,5}$ Doutora em Enfermagem. Professora da Graduação e do Programa de Pós-Graduação Mestrado em \\ Enfermagem da Universidade Federal do Piauí-UFPI. \\ ${ }^{3,4}$ Acadêmico do 90 período do curso de Bacharelado em Enfermagem da Universidade Federal do Piauí (UFPI). \\ E-mail: arthur_antunes03@hotmail.com
}

Cómo citar este artículo en edición digital: Luz, A. L. A; Luz, M. H. B. A; Antunes, A; Oliveira, G.S; Andrade, E.M.L.R; Miranda, S.M. (2014) Perfil de pacientes estomizados: revisão integrativa da literatura. Cultura de los Cuidados (Edición digital) 18, 39. Disponible en: http://dx.doi.org/10.7184/cuid.2014.39.13>

Correspondencia: Alyne Leal de Alencar Luz. Avenida Senador Helvídeo Nunes 4011, Junco, CEP: 64.607-755 Picos (PI). Correo electrónico: Alyne-luz@bol.com.br. Recibido: 01/04/2014; Aceptado: 14/06/2014

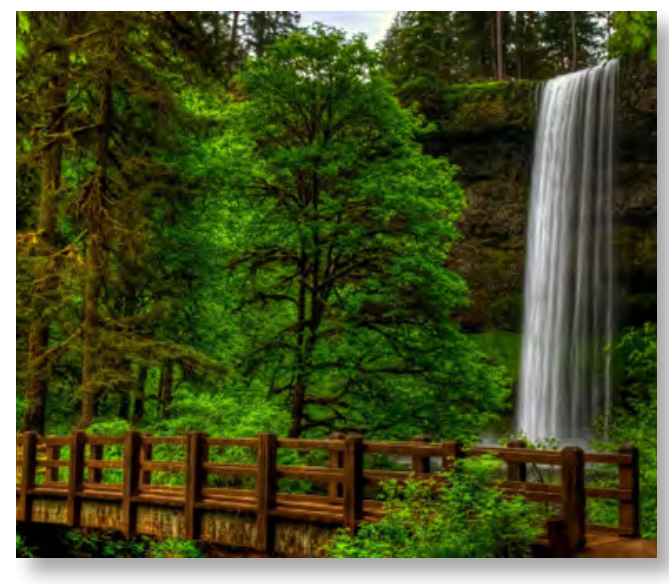

\section{ABSTRACT}

An analysis on the profile of ostomy, multicenter studies that portray the specific characteristics of these patients, contributes to meeting their real needs and the targeting of public policies and health to improve the quality of life for these clients. This study aims to investigate the scientific profile about ostomy patients in the period 2004-2012. It is an integrative literature review performed by an electronic search in databases: Lilacs, Scielo, and Bdenf Magazine estimated, because it is a specialized journal of the Brazilian Society of Stomatherapy. The sample consisted of ten articles which contemplated the inclusion criteria. It was found that all studies were conducted in Brazil in various states and regions and the vast majority of nurses. The sociodemographic and epidemiological prevalence of ostomy shows female predominance of older adults, colorectal cancer as the main cause for the realization of the stoma and colostomy as the predominant type of ostomy. It appears that the focus of this issue is still of scientific infancy, with specific studies, little comprehensive and low regularity. It is suggested that further studies be conducted to support improved health care user.

Key Words: Colostomy, Nursing, Health profile, Literature Review.

\section{RESUMO}

Uma análise acerca do perfil de estomizados, através de estudos multicêntricos que retratem as características específicas desses pa- 
cientes, contribui para o atendimento de suas reais necessidades e para o direcionamento de políticas públicas e de saúde para melhoria da qualidade de vida dessa clientela. Este estudo objetiva investigar a produção científica acerca do perfil de pacientes estomizados, no período de 2004 a 2012. Trata-se de uma revisão integrativa da literatura realizada por meio de busca eletrônica nas bases: Lilacs, Scielo, Bdenf e na Revista Estima, por tratar-se de um periódico especializado da Sociedade Brasileira de Estomaterapia. Fizeram parte da amostra dez artigos que contemplaram os critérios de inclusão. Identificou-se que todos os estudos foram realizados no Brasil, em estados e regiões diversas e a grande maioria por enfermeiros. $\mathrm{O}$ perfil sociodemográfico e epidemiológico dos estomizados mostra prevalência do sexo feminino, com predomínio de adultos e idosos, o câncer colorretal como principal causa para realização do estoma, e a colostomia como tipo de estomia predominante. Verifica-se que o foco desta temática ainda apresenta uma produção científica incipiente, com estudos pontuais, pouco abrangentes e baixa regularidade. Sugere-se que sejam realizados novos estudos para subsidiar uma melhor qualificação da assistência ao usuário.

Palavras-chave: Colostomia, Enfermagem, Perfil de saúde, Revisão da literatura.

\section{RESUMEN}

Mediante el análisis sobre el perfil de ostomia en los estudios multicéntricos que describen las características específicas de estos pacientes ostomizados, se contribuye a la satisfacción de sus necesidades reales y la focalización de las políticas públicas y de salud para mejorar la calidad de vida de lós mismos. Este estudio tiene como objetivo investigar el perfil científico de los pacientes ostomizados en el período 2004-2012. Se trata de una revisión integradora de la literatura realizada por una búsqueda electrónica en las bases de datos: Lilacs, scielo, Bdenf y la revista estima, porque es una revista especializada de la Sociedad Brasileña de estomaterapia. La muestra estuvo conformada por diez artículos que contemplan los criterios de inclusión. Todos los estudios se llevaron a cabo en Brasil en diversos estados y regiones y la gran mayoría de las enfermeras. La prevalencia sociodemográfica y epidemiológica de lós pacientes ostomizados muestra predominio femenino de adultos mayores, el cáncer colorrectal es la causa principal para la realización del estoma y colostomía como el tipo predominante de ostomía. Parece que el enfoque de este tema sigue aún en un período de relativaa infancia científica, con estúdios de escasa y baja regularidad. Se sugiere que otros estudios se llevaron a cabo con La finalidad de apoyar un mejor cuidado de la salud del usuario.

Palabras-clave: Colostomía, Enfermería, Perfil de salud, Revisión de la Literatura.

\section{INTRODUÇÃO}

A estomia é caracterizada como a exteriorização de um órgão interno para a superfície corporal, realizada por meio de ato cirúrgico, com a finalidade de desviar o trajeto fisiológico comprometido por alguma doença ou agravo. Possuem causas diversas e são classificadas conforme a finalidade do órgão afetado e designadas de acordo com o nome do segmento exteriorizado.

As estomias de eliminação intestinal são as mais frequentes, especialmente as colostomias, as quais são realizadas no intestino grosso e as ileostomias no intestino delgado. Podem ocorrer em todas as fases da vida, desde o neonato, 
crianças, adolescentes, adultos e principalmente os idosos, por patologias diversas, como as: anomalias congênitas, traumatismos abdominais, doenças inflamatórias intestinais: como a doença de Crohn, retocolite ulcerativa e diverticulite, assim como as neoplasias benignas e o predominantemente, câncer colorretal, entre outras (Macêdo; Nogueira; Luz, 2005).

Conforme a etiologia, condição clinica do paciente e circunstâncias envolvidas as estomias podem ser definitivas quando há impossibilidade de reestabelecimento do trajeto intestinal ou temporárias que são realizadas, geralmente com a finalidade de proteger uma anastomose e posteriormente, ser reestabelecido a continuidade do trânsito intestinal normal (Oliveira et al, 2010). A estomia de eliminação devido a inexistência do esfíncter, conduz o paciente à perda do controle voluntário das suas eliminações fisiológicas e dependência de equipamentos coletores denominados bolsas de estomias, situação esta, em que delineia uma denunciadora alteração da imagem corporal e auto-estima, o que pode configurar em uma mutilação sofrida, afetar o contexto social e a aptidão produtiva/laborativa (Barbutti; Silva; Abreu, 2008).

As necessidades de cuidados à pessoa estomizada caracteriza- se como um desafio para equipe multidisciplinar em sua abordagem terapêutica (Stumm; Oliveira; Kirschner, 2008). Essa condição implica, portanto, na busca de adequações em seu estilo de vida, que se reflete no aspecto fisiológico, psicológico e social e se traduz na relevância do processo de reabilitação a ser implementado a partir do diagnostico clinico, e na atenção continuada e permanente a fim de melhorar a qualidade de vida dessa clientela.

$\mathrm{O}$ enfermeiro, como integrante da equipe de saúde, tem como meta na sua atuação a sa- tisfação das necessidades de saúde do paciente, bem como a resolutividade dos problemas e manutenção da autonomia (Freitas, 2008). Nesse sentido, ressalta-se a necessidade de um cuidado integral, humanizado e sistematizado, com a implementação de estratégias de ensino, apoio e acompanhamento do autocuidado com a finalidade de promover e manter a saúde, evitar possíveis complicações e contribuir para reabilitação mediante a superação das dificuldades, conquista da autonomia, independência, reinserção e bem estar social, e principalmente, assegurar direitos de acesso aos serviços e garantia de boa qualidade de vida do estomizado.

Uma análise do perfil de estomizados, contribuirá para obtenção de dados de relevante importância pelo dimensionamento de suas características e necessidades para direcionar políticas de saúde e sociais, melhoria do desempenho técnico-científico dos profissionais que implica em intervenções efetivas assim como, fonte de informação e conhecimento para desenvolvimento de estudos futuros.

O presente estudo busca responder a seguinte questão: Qual o perfil sociodemografico e epidemiológico de pacientes estomizados? E tem como objetivo analisar o perfil de pacientes estomizados conforme a produção cientifica disponível em periódicos indexados em bases de dados nacionais e internacionais.

\section{METODOLOGIA}

Trata-se de uma revisão integrativa, a qual permite uma analise da literatura e contribui para síntese sobre métodos, resultados, discussão e conclusão de pesquisas relacionadas a um tema proposto (Mendes; Silveira; Galvão, 2008). Foi realizada conforme as etapas préestabelecidas: identificação do tema ou ques- 
tionamento da pesquisa, critérios de inclusão e exclusão dos estudos, definição das informações a serem extraída, avaliação dos estudos, interpretação dos resultados, apresentação da revisão/síntese do conhecimento.

A pesquisa ocorreu no período de maio a junho de 2013, sequencialmente nos bancos de dados: Literatura Latino Americana em Ciências da Saúde (LILACS), Scientifc Electronic Library On line (Scielo), Base de dados da enfermagem (Bdenf), com os seguintes descritores: ostomia, colostomia, perfil de saúde; e na Revista Estima por tratar-se de um periódico especializado da Sociedade Brasileira de Estomaterapia (SOBEST).

Os critérios de inclusão adotados foram: artigos publicados no período de 2004 a 2012, com resumo e texto completos disponíveis on line, idiomas em português ou espanhol, que retratassem no titulo, resumo e discussão, analise do perfil de pacientes estomizados. Critérios de exclusão: artigos encontrados em duplicidade e que não atenderam as questões e objetivos do estudo.

Para obtenção dos dados foi utilizado um formulário, baseado em instrumento previamente validado, adaptado pelos autores, o qual se constitui por informações gerais sobre os estudos selecionados para sintetizar as informações, como: base de dados, nome do periódico, titulo do artigo, primeiro autor, categoria profissional, ano de publicação, estado, região e país, delineamento da pesquisa e nível de evidencia dos estudos (Stetler et al, 1998). Assim como dados sociodemograficos dos estomizados: sexo, faixa etária, escolaridade, renda salarial e dados epidemiológicos: causa básica da estomia e tipo de estomia.

\section{RESULTADOS}

Foram encontrados no total, 45 estudos, dos quais 42 nas bases de dados: Lilacs (23),
Scielo (15) e Bdenf (4) e 3 na Revista Estima da SOBEST. Foram excluídos 35 por se encontrarem em duplicidade ou não corresponderem às questões norteadoras e objetivos propostos.

Desse modo, foram selecionados 10 artigos, sendo 5(50 \%) na lilacs, $1(10 \%)$ na Scielo, 1(10\%) na Bdenf e, 3(30\%) no periódico da SOBEST. Portanto, houve predominância de artigos na base de dados Lilacs e no periódico da SOBEST, com $80 \%$ dos estudos selecionado (Quadro 1).

Após leitura, análise e síntese dos 10 artigos selecionados e os dados produzidos mediante instrumento previamente elaborado, os estudos foram caracterizados conforme se observa no Quadro 2. Verifica-se que, considerando o periódico de publicação a metade (50\%) dos artigos encontram-se em revistas especializadas: $30 \%$ na Revista Estima e 20\% na Revista Brasileira de Coloproctologia, sendo a primeira vinculada a área de enfermagem e a segunda a área médica. Quanto aos demais periódicos, $10 \%$ na Revista Eletrônica de Enfermagem, 10\% na Texto \& Contexto em Enfermagem , 10\% em um periódico espanhol Gerokomos ,10\% na Scientia Medica e $10 \%$ na Cogitare Enfermagem. Quanto à categoria profissional dos autores verifica-se que a maioria dos artigos (90\%) foram realizados por enfermeiros e apenas $1(10 \%)$ por médicos.

Todos os estudos foram realizados no Brasil, embora um deles se encontre publicado em um periódico espanhol. Quanto à região geográfica, 30\% foram desenvolvidos na região sul do país, $30 \%$ na região nordeste, $20 \%$ na região sudeste, $10 \%$ na região norte e $10 \%$ na região centro-oeste.

Quanto a ano de publicação houve predomínio no período de 2007 a 2010, totalizando 08 artigos, distribuídos em duas publicações 
por ano, em seguida uma no ano de 2005 e outra em 2012. Porém, ressalta-se que não se encontrou nenhuma publicação nos anos de 2004, 2006 e 2011.

Em relação ao delineamento da pesquisa, todos os estudos (100\%) foram pesquisa de campo, original, com análise quantitativa dos dados, sendo em $50 \%$ a coleta de dados realizada por meio de pesquisas direta junto aos estomizados e 50\% mediante analise documental retrospectiva em prontuários. Quanto à avaliação do nível de evidencia, todos os estudos (100\%) apresentam nível IV, ou seja, estudos descritivos, com delineamento não experimental (Quadro 2).

Mediante aos aspectos sociodemográficos (Tabela 1), verificou-se que em relação ao gênero $60 \%$ dos estudos apontaram a predominância do sexo feminino. Quanto à faixa etária, as pesquisas se apresentam bastantes diversificadas em relação aos resultados, porem mediante analise, 70\% dos estudos apresenta a faixa etária predominante $\geq 40$ anos e $30 \%$ com a idade igual ou superior a 60 anos.

Quanto ao grau de escolaridade, a maioria (60\%) não apresentou esse registro para analise, porem $20 \%$ dos estudos apresentaram pacientes que predominantemente possuíam apenas o ensino fundamental completo, e os outros $20 \%$ o ensino fundamental incompleto. Ressalta-se que um estudo aponta igualdade dos resultados para analfabetos e ensino fundamental incompleto (Luz et al, 2009), porém os autores deste artigo, ressaltaram como critério o maior nível de escolaridade, ou seja, o ensino fundamental incompleto.

No tocante à renda salarial $40 \%$ dos estudos não apresentavam resultados referentes a variável, sendo que em $60 \%$ verifica-se uma predominância de poder aquisitivo entre um e três salário mínimos.
Tabela 1: Caracterização do perfil sociodemografico de pacientes estomizados conforme os artigos publicados de 2004 a 2012. ( $n=10)$

\begin{tabular}{|c|c|c|}
\hline Variável & n & $\%$ \\
\hline \multicolumn{3}{|l|}{ Sexo } \\
\hline Masculino & 4 & 40,0 \\
\hline \multirow[t]{2}{*}{ Feminino } & 6 & 60,0 \\
\hline & & Continua \\
\hline \multicolumn{3}{|l|}{ Faixa etária } \\
\hline$\geq 40$ anos & 7 & 70,0 \\
\hline$\geq 60$ anos & 3 & 30,0 \\
\hline \multicolumn{3}{|l|}{ Nivel de Escolaridade } \\
\hline Ensino fundamental completo & 2 & 20,0 \\
\hline Ensino fundamental incompleto & 2 & 20,0 \\
\hline Sem registro & 6 & 60,0 \\
\hline \multicolumn{3}{|l|}{ Renda salarial } \\
\hline 1-3 salários mínimos & 6 & 60,0 \\
\hline Sem registro & 4 & 40,0 \\
\hline
\end{tabular}

Quanto ao perfil epidemiológico, 80\% dos estudos selecionados evidenciaram a neoplasia maligna colorretal como principal etiologia para realização da estomia, 10 \% fatores clínicos e $10 \%$ não foi evidenciado a causa especificamente no estudo. Considerando o tipo de estomia predominante, todos os estudos (100\%) apontaram a colostomia.

\section{DISCUSSÃO}

Os estudos que abrangem essa temática apresentam características especificas conforme analise realizada, pois se verifica a grande diversidade distribuídas nas cincos regiões do território brasileiro, com predomínio na região sul e nordeste. Também ressalta-se a importância das publicações em revistas especializadas e a grande preocupação dos enfermeiros na investigação dos fatores sociodemograficos e epidemiológicos dos estomizados. Entretanto destaca-se que a falta de padroniza- 
ção em relação à faixa etária, limita a avaliação dos estudos, e outra característica de importância fundamental relaciona-se com a baixa amostra de pacientes submetidos às pesquisas realizadas.

Em análise aos estudos, observou-se que o paciente submetido a um processo de estomização apresenta determinadas peculiaridades em relação aos seus fatores sociodemograficos e epidemiológicos: predomínio do gênero feminino, idade avançada; assim como o baixo nível de escolaridade e renda salarial; a neoplasia maligna colorretal e a colostomia.

No tocante ao sexo, os estudos apontam o gênero feminino predominante para realização do estoma, assim como sua associação ao câncer colorretal, resultado que vem ao encontro com as estimativas de casos novos de câncer de colón e reto no Brasil em 2012 (Inca, 2012), que é de 14.180 casos em homens, correspondente a 15 casos novos a cada $100 \mathrm{mil}$, e 15.960 casos em mulheres, o que corresponde a 16 casos novos a cada 100 mil.

Entretanto estudos relatam que o câncer de colón incide na mesma proporção entre homens e mulheres, e que no segmento retal é mais frequente na população masculina, e que em relação a esse aspecto existem divergências, pois pesquisam evidenciam certa homogeneidade em relação ao sexo, mas demonstram a predominância do sexo masculino levando-se em conta as causas externas (Macêdo; Nogueira; Luz, 2005; Mantovani et al, 2007).

Em relação à faixa etária, os estudos demonstraram o predomínio de adultos e idosos, em que se verifica uma maior concordância entre as neoplasias e os estomizados com idade superior aos 40 anos tendo que o aumento da incidência pode ser exponencial, conforme o avanço da idade, de tal forma que o envelhecimento apresenta-se como um fator de risco

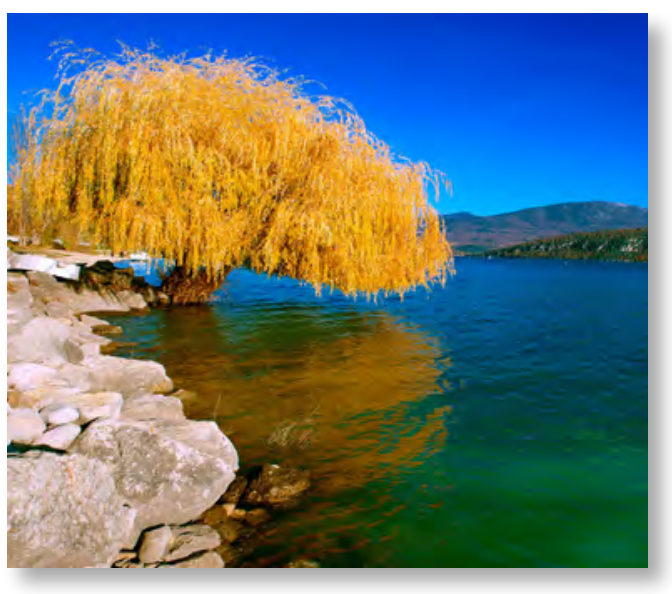

para o desenvolvimento da neoplasia e consequentemente a possibilidade de realização do estoma.

O critério socioeconômico conjuntamente com o nível de escolaridade tem importância significativa na analise do perfil dessa clientela, pois são determinantes sociais da saúde e relacionam- se com o contexto social e politicas públicas voltadas ao estomizado. $\mathrm{O}$ baixo poder aquisitivo evidenciado neste estudo vai influenciar nos níveis de saúde do paciente submetido a uma estomização, pois poderão apresentar dificuldades para adquirir dispositivos coletores e adjuvantes imprescindíveis para uma boa qualidade de vida, e de extrema importância para o processo reabilitatório do paciente (Fernandes; Miguir; Donoso, 2010; Silva; Silva; Cunha, 2012). Entretanto cabe destacar, o papel dos profissionais de saúde, em especial o enfermeiro no sentido de encaminhar formalmente os pacientes aos polos de distribuição de materiais oferecidos pelo Sistema Único de Saúde (SUS).

O baixo nível de escolaridade se torna um dado preocupante, pois quanto menor o grau de instrução maior é a dificuldade de acesso ao conhecimento a cerca do processo da doença, tendo que o paciente se torna sujeito passivo, alienado e não questionador de seu tratamento 
Ao analisar a principal causa patológica associada ao estoma, a neoplasia maligna colorretal, mostrou- se coerente aos elevados índices de pacientes com estomias intestinais, pois abrange tumores do intestino grosso (colon) e o reto (Violini; Mathias; Uchimura, 2008), sendo que maioria dos casos que envolvem a intervenção cirúrgica no tratamento do câncer colorretal resultam consequentemente na formação da colostomia , segmento do colón do intestino grosso classificado como o tipo de ostoma exteriorizado que ocorre com maior frequência, com o objetivo de eliminação fecal.

Estudo aponta a região do sigmoide e o colón descendente como os locais mais frequentes para o surgimento do câncer, seguidos do colón ascendente e transverso (Macêdo; Nogueira; Luz, 2005). É importante ressaltar que a causa também pode estar relacionado com o comportamento e o contexto social populacional, pois pesquisa que envolve o cenário de emergência apresentam como causa básica para realização do estoma, os traumas e a violência (Luz et al, 2009).

Contudo ressalta- se a extrema importância da equipe multidisciplinar, em especial o enfermeiro, que atua no processo de reabilitação, tendo que adequar-se a uma linguagem acessível de tal forma a favorecer o autocuidado e segurança do paciente como fator preponderante em sua recuperação. Estudo de Moraes et al (2009) evidencia que praticamente 50\% dos pacientes que se submeteram ao processo de estomização necessitam de cuidados específicos, de auxilio em relação ao autocuidado e acompanhamento interdisciplinar; o trabalho mostrou o enfermeiro como profissional habilitado a proporcionar um cuidado eficiente, favorecendo o processo de autonomia e reabilitação do paciente.
De maneira conjunta com a equipe interdisciplinar na elaboração dos planos de cuidados, o enfermeiro visa às orientações ao paciente sobre uma melhor compreensão da estomia, seu tratamento, autocuidado com a finalidade de evitar possíveis complicações, como: dermatite periostomal, retração, prolapso, necrose isquêmica, entre outras (Santos et al, 2007; Souza et al, 2010), e busca resoluções priorizando a esfera física, psicossocial e emocional, que juntamente com o suporte familiar terão influencia significativa no processo de reabilitação do paciente estomizado.

\section{CONCLUSÃO}

Verifica-se que o foco desta temática ainda apresenta uma produção científica incipiente, com estudos pontuais, pouco abrangentes e baixa regularidade. O que implica a necessidade dos profissionais, em especial o enfermeiro que lida diretamente com o paciente, levantar questões que contribuam para uma politica de implementação nacional a esse grupo especifico de pacientes.

Diante disso, torna-se imperioso evidenciar analises sociodemograficas e epidemiológicas, de tal modo a fornecer subsídios, conhecimentos e com a finalidade de traçar o planejamento da assistência, com incorporação dos fundamentos necessários aos profissionais das características fundamentais dos clientes estomizados.

\section{REFERÊNCIAS BIBLIOGRÁFICAS}

- Barbutti, R.C.S; Silva, M.C .P; Abreu, M.A.L. (2008) Ostomia, uma difícil adaptação. Rev. SBPH. 11(2): 27-39.

- Fernandes, R.M; Miguir, E.L.B; Donoso, T.V. (2010) Perfil da clientela estomizada residente no município de ponte nova, Minas gerais. Rev. Bras. Coloproct. 30(4): 385-92.

- Freitas, G. F. (2008) Bioética no cuidado e direitos dos pacientes e familiares. Cultura de los cuidados. Año XII 
(24): 75-81. Disponível em: <http://culturacuidados. ua.es/enfermeria/article/view/308>. Acesso em: 06 Nov. 2013

- Inca. Instituto Nacional do Câncer; Ministério da saúde. Estimativa 2012: incidência de câncer no Brasil; 2013. [ acessado em 2013 jun 27 ]. Disponível em: http://www. inca.gov.br/estimativa/2012/ index. asp?ID=5.

- Luz, M.H.B.A. et al. (2009) Caracterização dos pacientes submetidos a estomas intestinais em um hospital publico de Teresina-PI. Texto \& contexto enferm. 18(1): 140-6.

- Macêdo, M.S; Nogueira, L.T; Luz, M.H.B.A. (2005) Perfil dos estomizados atendidos em Hospital de referencia em Teresina. Revista Estima. 3(4): 25-8.

- Mantovani, M.F. et al. (2007) O perfil dos usuários cadastrados na Associação Paranaense de estomizado - APO. Cogitare enferm. 12(1): 76-81.

- Mendes, K.D; Silveira, R.C.C.P; Galvão, M.C. (2008) Revisão Integrativa: método de pesquisa para a incorporação de evidências na saúde e na enfermagem. Texto \& contexto enferm. 17(4): 758-64.

- Moraes, J.T. et al. (2009) Caracterização dos estomizados atendidos pela secretaria municipal de saúde de Divinópolis- MG. Revista Estima. 7( 3): 31-37.
- Oliveira, G. et al. (2010) Impacto da ostomia: sentimentos e habilidades desenvolvidas frente à nova condição de vida. Revista Estima. 8(1): 19-25.

- Santos, C.H.M. et al. (2007) Perfil dos pacientes ostomizado e complicações relacionadas ao estoma. Rev. Bras. Coloproct. 27(1): 16- 9.

- Silva, A.C; Silva, G.N.S; Cunha, R.R. (2012) Caracterização das pessoas estomizadas atendidas em consulta de enfermagem em serviço de estomaterapia do município de Belém- PA. Revista Estima. 10(1): 12-9.

- Souza, A.P.M.A. et al. (2010) Perfil clínico-epidemiológico de los pacientes atendidos y censados en el Centro Paraibano de Ostomizados- João Pessoa, Brasil. Gerokomos. 21(4): 183-90.

- Stetler, C.B. et al. (1998) Utilization-focused integrative reviews in a nursing service. Appl Nurs Res. 11(4): 195206.

- Stumm, E.M.F; Oliveira, E.R.A; Kirschner, R.M. (2008) Perfil de pacientes estomizados. Sci. med. 18(1): 26-30.

- Violini, M.R; Mathias, T.A.F; Uchimura, T.T. (2008) Perfil de clientes colostomizados inscritos em programa de atenção ao estomizado. Rev. Eletrônica Enferm. 10(4): 924- 32 .

Quadro 1: Distribuição dos estudos encontrados e selecionados, segundo as bases de dados.

\begin{tabular}{|c|c|c|c|}
\hline BASE DE DADOS & $\begin{array}{c}\text { ARTIGOS } \\
\text { ENCONTRADOS }\end{array}$ & $\begin{array}{c}\text { ARTIGOS } \\
\text { SELECIONADOS }\end{array}$ & $\%$ \\
\hline LILACS & 23 & 5 & $50 \%$ \\
\hline SCIELO & 15 & 1 & $10 \%$ \\
\hline BDENF & 4 & 3 & $10 \%$ \\
\hline SOBEST & 3 & 10 & $100 \%$ \\
\hline TOTAL & 45 & & $30 \%$ \\
\hline
\end{tabular}


Quadro 2: Caracterização dos artigos selecionados conforme as variáveis do estudo.

\begin{tabular}{|c|c|c|c|c|}
\hline $\begin{array}{l}\mathrm{N}^{0} \mathrm{de} \\
\text { ordem }\end{array}$ & Periódico & Titulo & ado/Região/Pais/Ano & $\begin{array}{l}\text { Delineamento } \\
\text { da pesquisa }\end{array}$ \\
\hline 01 & $\begin{array}{l}\text { Revista } \\
\text { Estima }\end{array}$ & $\begin{array}{l}\text { Perfil dos estomizados } \\
\text { atendidos em hospital de } \\
\text { referência em Teresina }\end{array}$ & $\begin{array}{l}\text { Piaui/ Nordeste } \\
\text { Brasil/ } 2005\end{array}$ & $\begin{array}{c}\text { Estudo } \\
\text { quantitativo/ } \\
\text { pesquisa de campo }\end{array}$ \\
\hline 02 & $\begin{array}{l}\text { Rev. Bras. } \\
\text { Coloproct. }\end{array}$ & $\begin{array}{l}\text { Perfil de paciente } \\
\text { ostomizado e complicações } \\
\text { relacionadas ao estoma }\end{array}$ & $\begin{array}{l}\text { Mato grosso do sul/ } \\
\text { centro- oeste } \\
\text { Brasil/2007 }\end{array}$ & $\begin{array}{c}\text { Estudo } \\
\text { quantitativo/ } \\
\text { documental }\end{array}$ \\
\hline 03 & $\begin{array}{l}\text { Cogitare } \\
\text { Enferm. }\end{array}$ & $\begin{array}{l}\text { Perfil dos usuários } \\
\text { cadastrados na associação } \\
\text { Paranaense de ostomizado }\end{array}$ & $\begin{array}{l}\text { Paraná/Sul } \\
\text { Brasil/2007 }\end{array}$ & $\begin{array}{l}\text { Estudo } \\
\text { quantitativo/ } \\
\text { documental }\end{array}$ \\
\hline 04 & $\begin{array}{l}\text { Scientia } \\
\text { Medica }\end{array}$ & $\begin{array}{l}\text { Perfil de paciente } \\
\text { ostomizado }\end{array}$ & $\begin{array}{l}\text { Rio grande do Sul/ } \\
\text { Sul/Brasil/2008 }\end{array}$ & $\begin{array}{l}\text { Estudo quantitativo } \\
\text { documental }\end{array}$ \\
\hline 05 & $\begin{array}{c}\text { Rev Eletr. } \\
\text { Enf: }\end{array}$ & $\begin{array}{l}\text { Perfil de clientes } \\
\text { colostomizados Inscritos } \\
\text { em um programa } \\
\text { de atenção ao } \\
\text { estomizado }\end{array}$ & $\begin{array}{l}\text { Paraná/ Sul / } \\
\text { Brasil/2008 }\end{array}$ & $\begin{array}{l}\text { Estudo quantitativo/ } \\
\text { documental }\end{array}$ \\
\hline 06 & $\begin{array}{l}\text { Texto\& } \\
\text { Contexto } \\
\text { Enf: }\end{array}$ & $\begin{array}{l}\text { Caracterização dos pacientes } \\
\text { submetidos estomas } \\
\text { intestinais em um } \\
\text { hospital Publico de } \\
\text { Teresina-PI }\end{array}$ & $\begin{array}{l}\text { Piaul/ Nordeste } \\
\text { Brasil/ } 2009\end{array}$ & $\begin{array}{c}\text { Estudo } \\
\text { quantitativo/ } \\
\text { pesquisa de campo }\end{array}$ \\
\hline 07 & $\begin{array}{l}\text { Reyista } \\
\text { Estima }\end{array}$ & $\begin{array}{l}\text { Caracterização dos estomizados } \\
\text { atendidos pela secretaria } \\
\text { Municipal de saủde de } \\
\text { Divinópolis-MG }\end{array}$ & $\begin{array}{l}\text { Minas Gerais/sudeste } \\
\text { Brasil/ } 2009\end{array}$ & $\begin{array}{c}\text { Estudo } \\
\text { quantitativo/ } \\
\text { pesquisa de campo }\end{array}$ \\
\hline 08 & $\begin{array}{l}\text { Rev. Bras. } \\
\text { Coloproct. }\end{array}$ & $\begin{array}{l}\text { Perfil da clientela estomizada } \\
\text { residente no municipio de } \\
\text { Ponte Nova, Minas Gerais }\end{array}$ & $\begin{array}{l}\text { Minas Gerais/sudeste } \\
\text { Brasil/ 2010 }\end{array}$ & $\begin{array}{c}\text { Estudo } \\
\text { quantitativo } \\
\text { pesquisa de campo }\end{array}$ \\
\hline
\end{tabular}

